\title{
Xihmai
}

Universidad La Salle Pachuca

xihmai@lasallep.edu.mx

Teléfono: 01(771) 7170213 ext. 1406

Fax: 01(771) 7170309

ISSN (versión impresa):1870_6703

México

Selen Catalina Arango Rodríguez

LAS TRANSFORMACIONES DE LA IDEA DE EXPERIENCIA FEMENINA EN GLORIA ANZALDÚA

TRANSFORMATIONS OF THE IDEA OF FEMALE EXPERIENCE IN

GLORIA ANZALDÚA

Xihmai, año 2017/vol. XII, número 23

Universidad La Salle Pachuca

pp. $29-44$ 
Selen Catalina Arango Rodríguez Las Transformaciones de la Idea de Experiencia Femenina en Gloria Anzaldúa

Revista Xihmai XII (23), 29-44, Enero - junio 2017

Xihmai 30 
Las Transformaciones de la Idea de Experiencia Femenina en Gloria Anzaldúa

Revista Xihmai XII (23), 29-44, Enero - junio 2017

\section{LAS TRANSFORMACIONES DE LA IDEA DE EXPERIENCIA FEMENINA EN GLORIA ANZALDÚA}

\section{TRANSFORMATIONS OF THE IDEA OFFEMALE EXPERIENCE IN \\ GLORIA ANZALDÚA}

Selen Catalina Arango Rodríguez

Doctora en Pedagogía de la Universidad Nacional Autónoma de México. Docente e integrante del Grupo de Investigación FORMAPH de la Facultad de Educación y coordinadora de la Maestría en Educación de Profundización de la Universidad de Antioquia, Colombia. selen.arango@outlook.com

\section{Recibido 8-07-16 Aceptado 25-07-16 Corregido 14-08-16}

\section{Resumen}

La obra de Gloria Anzaldúa, en la actualidad, tiene una gran importancia para los estudios latinoamericanos y para la crítica literaria feminista. Esto se debe a la influencia de los estudios acerca de la interseccionalidad en el feminismo con las coordenadas de sexo, género, raza y clase social, y de los cambios en la crítica literaria interesada en estudiar la creación literaria en clave de políticas de la escritura. También porque a partir de los 80 se hizo más visible la producción literaria de las mujeres y surgió un marcado interés en el estudio del sujeto del feminismo. Los anteriores hitos llevaron a las feministas a preguntarse por el sujeto del feminismo, y con él por la experiencia de este sujeto. Se encontró que la experiencia es una construcción como el género de los sujetos y que, por lo tanto, no puede adjudicarse un tipo de experiencia en razón al sexo biológico, la raza y la clase social. Este artículo, a partir de la obra de Gloria Anzaldúa, describe las transformaciones de la idea de experiencia en el feminismo y la aborda como una construcción e interpretación de sí mediante políticas de la escritura.

\section{Palabras claves:}

Gloria Anzaldúa, experience, subject of feminism, feminist literary criticism, politics of writing. 


\section{Sumary:}

The work of Gloria Anzaldúa is currently of great importance for Latin American studies and for feminist literary criticism. This is due to the influence of intersectionality studies in feminism with the coordinates of sex, gender, race and social class, and of changes in literary criticism interested in studying literary creation in the key of writing policies. Also because from the 80's the women's literary production became more visible and there was a marked interest in the study of the subject of feminism. The previous milestones led feminists to wonder about the subject of feminism and the experience of this subject. It was found that experience is a construction as the gender of subjects and therefore can not be awarded a type of experience on the grounds of biological sex, race and social class. This article, based on the work of Gloria Anzaldúa, describes the transformations of the idea of experience in feminism and approaches it as a construction and interpretation of itself through policies of writing.

\section{Key notes:}

Experience, Subject of feminism, Writing policies, Gloria Anzaldúa

La experiencia femenina comúnmente se asocia a las mujeres. Sin embargo, al indagar en la teoría feminista puede identificarse que la noción de experiencia requiere abordarse como el feminismo retoma la idea de su sujeto: mediante una interpretación. Tanto el sujeto de la experiencia como el del feminismo no existen, ni pueden identificarse con un grupo humano.

En los años ochenta del siglo veinte y en el marco de la epistemología feminista, emerge una preocupación por el sujeto del feminismo. Al cuestionar la naturalización de la experiencia femenina como propia de las mujeres, los/as autores/as comenzaron a preguntarse por el sujeto del feminismo como una construcción discursiva y socialmente localizada (Bondi, 1996; Williams, 1994).

Dentro de este contexto, en 1980 Gloria Anzaldúa publica el escrito Hablar en lenguas. Una carta a escritoras tercermundistas, en el cual insiste a sus hermanas de color, a las lesbianas, a las mujeres latinas, asiáticas, no dejar de escribir así lo hagan en lenguas no entendidas por sujetos que no han reconocido su diferencia. Su manera de escribir es la de las mujeres que (Anzaldúa 1988, p. 223) "sabíamos que éramos diferentes, apartadas, exiliadas de lo que se considera "normal', blanco-correcto." Su obra convoca a las Xihmai 32 
mujeres cuyas palabras cuestionan la idea de la experiencia femenina como una esencia.

Experiencia (1992) de Joan Scott es un texto fundacional para reflexionar acerca del sujeto del feminismo y la experiencia. En este artículo Scott (1992, p. 53) plantea que la experiencia no es interna o externa, objetiva o subjetiva, o un discurso cuya intención es establecer cualidades de los individuos mediante la naturalización de "“categorías tales como hombre, mujer, negro, blanco, homosexual y heterosexual, al tratarlas como características dadas de los individuos". Para la autora, los individuos no tienen la experiencia: son construidos por ella. La experiencia para Scott $(1992$, p. 66) es (...) un evento lingüístico (no ocurre fuera de significados establecidos), pero tampoco está confinada a un orden fijo de significado. Ya que el discurso es por definición compartido, la experiencia es tanto colectiva como individual. La experiencia es la historia de un sujeto. El lenguaje es el sitio donde se representa la historia. La explicación histórica no puede, por lo tanto, separarlos.

La experiencia, al ser un evento discursivo y por tanto, del orden del lenguaje, puede ser analizada a partir de los significados que produce. Estos significados generan al sujeto. Analizar la experiencia es también analizar la historia de la formación del sujeto; y la historia del sujeto no pude ser interpretada sin ser comprendida como resultado de diferentes discursos y sin una localización específica. Esa historia de la formación no es otra que una historia de las experiencias, la cual no puede ser contada de manera lineal sino en forma de una trayectoria de la incertidumbre, situada y en proceso de transformación permanente.

\subsection{Primera transformación: situar el lugar desde donde se observa}

"Soy una artista del tercer mundo", afirma la artista Doris Salcedo en una entrevista televisiva. Con estas palabras sitúa su forma de mirar, que le permite producir obras de arte en unas coordenadas y circunstancias específicas. Donna Haraway (1995) en Conocimientos situados: la cuestión científica en el feminismo y el privilegio de la perspectiva parcial se pregunta por la manera como miramos y la pretensión de objetividad mientras lo hacemos, propia del discurso científico. Esta mirada es realizada por un sujeto, generalmente masculino, con preferencias de ser o no visto, con el convencimiento de representar algo o alguien mientras imagina que no deja huellas de cómo se representa, de cómo se ve a sí mismo. Para Haraway (1995, p. 324), esta 
objetividad en el marco del feminismo, debe desarrollar proyectos basados en una ciencia feminista que los haga "(...) paradójicos y críticos: la objetividad feminista significa, sencillamente, conocimientos situados".

Los conocimientos situados se refieren al acto de ser consciente de hacia dónde se mira, hacia quiénes y desde dónde mientras nos sabemos observadas. Para Haraway, necesitamos traer aquello que vemos hacia nosotras mismas y definir si estamos o no en ese tipo de experiencia que se espera de nosotras. Situar la experiencia es tomar responsabilidad ante lo que observamos o se nos pide observar; estar sujetos a ser transformadas en el acto de conocer en un tiempo y lugar determinados.

Esta mirada también se sitúa desde abajo. Gloria Anzaldúa en Borderlands/La frontera. The New Mestiza (1987) entrega un hito para los estudios feministas interesados en investigar la experiencia en el marco de nuestra realidad americana que para Anzaldúa es fronteriza y sin adscripción a un territorio específico por derecho de nacimiento. La fuerza de este texto resignifica la carga negativa de la palabra "mestiza". Trasladó la acción de nombrar con esta palabra a las/os hijas/os de los españoles/as con indígenas en el periodo de la colonización, hacia los estudios acerca de la identidad de las/os chicanas/os (aquellas personas nacidas en los Estados Unidos de padres y madres mexicanas o con ascendencia mexicana). Ser mestiza significa sentirse honrada/o por sus ancestros mexicanos. Resituar la palabra "mestiza" en el contexto de la frontera propone un reconocimiento de ida y vuelta, un intercambio entre sujetos de diferentes clases sociales, razas y géneros, dentro de un espacio que no solo divide a dos territorios sino en el que también le suceden experiencias a los sujetos.

La frontera de México con los Estados Unidos es habitada por lo prohibido y lo olvidado. Habitada por quienes añoran una mejor vida, pero que constantemente son expulsados/as de ella y señalados/as como sujetos a quienes se les impide tal búsqueda por su raza, sexo y/o clase social. Las personas que encarnan y experimentan lo prohibido y lo olvidado son los hombres y las mujeres del tercer mundo, morenos, pobres, indígenas o con ascendencia prehispánica, sin una lengua 'oficialmente aceptada', y/o con sexualidades no heterosexuales.

El trabajo de Anzaldúa irrumpe en la construcción de las fronteras para demostrar que con una frontera no solo se marcan límites geográficos sino Xihmai 34 
también culturales e identitarios. La experiencia en el trabajo de Alzaldúa es la de la frontera, la cual no corresponde a la fragmentación del límite. Es la experiencia de posicionarse desde abajo, en la posición de la crítica, desde donde pueden observarse los pliegues y contrastes de la diferencia, de la pobreza, el lugar del caminante, del viajero a pie. La obra de Anzaldúa hace parte del feminismo de los 80, interesada en cuestionar la idea esencialista de una experiencia femenina para localizarla discursivamente.

Lynda Alcoff (1988), como Scott (1992), asume la experiencia como efecto de discursos, que caracteriza como hegemónicos, y que guardan diferentes formas y efectos. Al retomar los planteamientos de Lauretis en Alicia ya no (1984, p. 253), Alcoff arguye la imposibilidad de establecer una "experiencia como femenina" o "masculina" en tanto la experiencia: (...) es el proceso por el cual se construye la subjetividad para todos los seres sociales. A través de ese proceso uno se ubica o es ubicado en la realidad social y de ese modo percibe y comprende como subjetivas (referidas a y originadas en uno mismo) esas relaciones - materiales, económicas e interpersonales - que de hecho son sociales y, en una perspectiva más amplia, históricas (Cursivas de la autora).

Como escribe de Lauretis, este proceso entiende que la subjetividad no tiene un punto de partida o una meta a la cual llegar, sino que es una manera de interactuar y dar significación a la realidad social. La experiencia, escribe la autora italiana (1994, p. 253), posibilita que con nuestro compromiso personal, subjetivo, dialoguemos con ideales o prescripciones externas, participemos en las actividades, discursos e "(...) instituciones que dotan de importancia (valor, significado, y afecto) a los acontecimientos del mundo".

Teresa de Lauretis $(1989$, p. 8) argumenta que el género no es una capacidad, sino "el conjunto de efectos producidos en los cuerpos, los comportamientos y las relaciones sociales". Esta consideración parte de Foucault, quien escribe en el primer tomo de la Historia de la sexualidad, que la sexualidad es un punto de pasaje de las relaciones de poder. No obstante, de Lauretis $(1989$, p. 9) acerca el trabajo de Foucault hacia los estudios del género al decir que el filósofo francés ignoró "las conflictivas investiduras de varones y mujeres en los discursos y las prácticas de la sexualidad, [y excluyó], si bien no impide, la consideración del género". Por lo tanto, el género (de Lauretis, 1989, p. 8) es producto de varias "tecnologías sociales y de discursos institucionalizados, de epistemologías y de prácticas críticas de la vida cotidiana". Tecnologías, prácticas y discursos que hacen parte de un sistema de sexo-género que 
preexiste al individuo. Un sistema que prescribe lo que se entiende en cada cultura como femenino y como masculino, que asigna una identidad y prestigios según el género, y otorga ubicaciones en la jerarquía social.

El género no es un punto de partida, sino un constructo que se conforma en interesantes posibilidades de significación con las que el sujeto se encuentra en su cultura y se identifica. Por su parte, la experiencia constituye semiótica e históricamente la subjetividad y esto se debe a que el sujeto y la realidad social también tienen una naturaleza semiótica. En este sentido, Teresa de Lauretis (1984, p. 289) lanza la siguiente pregunta: “ ¿es el sujeto femenino un sujeto que se constituye en un tipo particular de experiencia, específicamente a través de una experiencia particular de la sexualidad?" y plantea la siguiente respuesta:

Y si respondemos que sí, que una cierta experiencia de la sexualidad produce un ser social al que podemos llamar sujeto femenino; si es esa experiencia, ese complejo de hábitos, disposiciones, asociaciones y percepciones lo que engendra a uno como femenino, entonces eso es lo que debe analizar, comprender, articular la teoría feminista.

El género tiene mucho que ver con la experiencia en tanto no es una identidad original sino una construcción que da cuenta de su representación y de su propio proceso. Así como la experiencia comparte con el viaje la cualidad de la incertidumbre (no saber si se llegará a ser "una mujer" o "un hombre" determinado), el género comparte con la identidad la cualidad de ser un proceso, un "estando" o un "siendo", diría Jenkins (1996). Entender el género como una construcción social y cultural allana el terreno para afirmar que la experiencia tampoco puede ser considerada como "verdadera," -es verosímil, asemeja una verdad inamovible- y la inexistencia de una experiencia que sea femenina estructuralmente. El sistema de verosimilitud es sostenido por las intenciones de un sistema de sexo-género para conseguir y perpetuar unos ideales de formación femeninos en una cultura específica, a través de señalamientos y tradiciones de lo que "debe ser una mujer".

\subsection{Segunda transformación: la política de la escritura de la propia experiencia}

El proceso de la escritura es una construcción. Es la oportunidad para que los sujetos creen su propia historia y responda a preguntas que interpelen su experiencia. Gloria Anzaldúa (1988, p. 220 y 221) asimiló la escritura como un acto que le permitía responder a los interrogantes que la sociedad Xihmai 36 
estadounidense se planteaba frente a la posibilidad de que una mujer chicana, mestiza, pudiese escribir.

¿Quién nos dio el permiso de realizar el acto de escribir? ¿Por qué será que el escribir se siente tan innatural para mí?

¿Quién soy yo, una pobre Chicanita del campo, que piensa que puede escribir? (Cursivas de la autora).

¿Qué tenemos para contribuir, para dar? (...) ¿Acaso no nos dice nuestra clase, nuestra cultura, tanto como el hombre blanco que el escribir no es para mujeres tal como nosotras?

Siguiendo con de Lauretis (1984), el proceso a través del cual el "yo" interpreta un signo resulta de un hábito en el marco de una realidad social específica. Por ejemplo, la relación del color rosa en las sociedades machistas con lo femenino, es producto de un hábito adquirido. Tal hábito hace que el "yo" actúe de acuerdo al signo, y que al ver a una persona con una camisa rosa se considere que debe recibir un trato de mujer. Teresa de Lauretis (1984, p. 289) apropia la noción de "Hábito" de Pierce y la iguala a experiencia: Hábito=Experiencia, como

(...) actitud "energética", como disposición somática a la vez abstracta y concreta, forma cristalizada de un esfuerzo muscular/mental previo, sugiere poderosamente a un sujeto alcanzado por la actividad de los signos, a un sujeto físicamente implicado o corporalmente comprometido en la producción de significado, en la representación y en la autorepresentación"

La escritura es la afirmación de la experiencia de los sujetos. Con ella producen otras significaciones a los signos aceptados socialmente como "femeninos" o "masculinos". Este trabajo se ancla a la experiencia de su formación, por lo que su escritura se dedica a interpretar, analizar y entender los hábitos con los cuales fueron formados.

Con su obra, Anzaldúa cuestiona que las mujeres y los sujetos feminizados (homosexuales, hombres y mujeres pobres, indígenas, campesinos/as) solo poseen saberes, historias orales y testimonios. La experiencia de la escritura en de Anzaldúa es el empeño por la representación de la fractura y la crisis

\footnotetext{
${ }^{1}$ El término "hábito" de Lauretis (1984, p.276) lo retoma de Pierce quien lo usa en su sentido más amplio y que "(...) designa una especialización tal, original o adquirida, de la naturaleza de un hombre, o de un animal, o de un vino, o de una sustancia química cristalizable, o de cualquier otra cosa, de tal forma que se comportarán o tenderán a comportarse siempre de una forma describible en términos generales en toda ocasión (en una parte considerable de las ocasiones) que puede presentarse en un carácter describible en términos generales".
} 
propia en una sociedad heteronormativa y heterosexual donde "lo femenino" parece tener mayores dificultades para obtener privilegios políticos.

La práctica de la escritura, es una práctica de la concienciación. En palabras de Lauretis (1984), la concienciación, refiriéndose a las mujeres, es la forma a través de la cual pueden acceder a la interpretación de su experiencia y al reconocimiento de cómo ésta las constituye.

La escritura necesita de un cuarto propio como lo escribió Virginia Woolf: “... para escribir novelas, es necesario que una mujer cuente con dinero y con un cuarto propio" (2006, p. 7), y con este cuarto se refiere a que tengamos derecho a unas condiciones básicas como un buen salario y una casa en la que además de los espacios familiares, si es el caso, tengamos un cuarto donde podamos realizar nuestras lecturas previas a la escritura.

Pero este cuarto propio es solo para algunas mujeres, generalmente, de una clase social alta y/o acomodada que no les exige renunciar a su oficio de escritoras para buscar un trabajo que les signifique algún reconocimiento económico. Las otras mujeres, los otros que no pueden tener este cuarto propio ¿qué políticas de la escritura han creado?

Gloria Anzaldúa dirige una carta a las escritoras tercermundistas en 1980. En esta carta les invita a escribir por fuera del cuarto propio de Woolf: en todos los lugares donde podamos hacerlo; así seamos "mujeres de color" en un mundo donde "lo blanco", heterosexual, cuenta con posibilidades económicas y buenas relaciones sociales para ser lo más publicado y leído. Anzaldúa, al reconocer la dificultad que le dio alejarse del discurso académico para escribir una carta, instaura una política del reconocimiento de la escritura testimonial, de la escritura de la intimidad situada e interceptada por las condiciones de sexo, género, color y clase.

Sabe de las condiciones que influyeron para la emergencia de una política de la escritura fuera del cuarto propio: los intentos por exterminar una lengua a través de la imposición hegemónica (lo que se ha nombrado como idioma nacional), las estrategias de deslegitimación a través de la enseñanza de una "lengua oficial" diferente de la lengua materna, el caso de la imposición del inglés en los Estados Unidos a estudiantes chicanos/as a través del señalamiento del español como una lengua inferior; o los casos de mujeres lesbianas, que con hijos/as y separadas de sus esposos, son señaladas por su sociedad y casi no tienen tiempo para escribir; o los hombres que quieren Xihmai 38 
alternar el trabajo en el cuidado de los animales y de la tierra con la escritura; o una mujer indígena que desea recorrer las poblaciones contando historias, sin casarse.

Estas condiciones son el punto de partida para una política de la escritura de la experiencia, porque la escritura es el lugar que salva del tiempo de complacimiento con la realidad tal y como está. Anzaldúa (1980, p. 224-225) sigue diciendo:

Olvídate del "cuarto propio" -escribe en la cocina, enciérrate en el baño. Escribe en el autobús o mientras haces fila en el Departamento de Beneficio Social o en el trabajo durante la comida, entre dormir y estar despierta. Yo escribo hasta sentada en el excusado. No hay tiempos extendidos con la máquina de escribir a menos que seas rica o tengas un patrocinador (puede ser que ni tengas una máquina de escribir). Mientas lavas los pisos o la ropa escucha las palabras cantando en tu cuerpo. Cuando estés deprimida, enojada, herida, cuando la compasión y el amor te posean. Cuando no puedas hacer nada más que escribir.

Escribir para ordenar el mundo desde la propia mirada, así sea en los tiempos y espacios no esperados por la escritura. La escritura para Anzaldúa es un oficio al cual se es convocada/o; a ella la convoca su condición de mujer tercemundista, de chicana, de lesbiana. Escribe porque no le importan las expectativas que los otros se hagan respecto a su escritura: ella es otra más que da testimonio de su condición. Escribe porque a las mujeres las exiliaron de la palabra escrita, de la creación literaria y cuando lo hacen algunos/as increpan su acto al decir que enseñan al público su vida privada. Escribe porque no quiere afirmar que su experiencia sea más difícil o mejor que las de otras mujeres, y por la necesidad de no trivializar unas experiencias ante la de ella. Cuenta a través de un tejido que amarra los intentos para deslegitimar su voz (Anzaldúa, 1980, p. 224).

El peligro de escribir es no fundir nuestra experiencia personal y nuestra perspectiva del mundo con la realidad social en que vivimos, nuestra historia, nuestra economía, y nuestra visión. Lo que nos valoriza a nosotras como seres humanas nos valoriza como escritoras. No hay tema que sea demasiado trivial. El peligro es ser demasiado universal y humanitaria e invocar lo eterno para el sacrificio de lo particular y de lo femenino y el momento histórico específico.

Al pensar en políticas de la escritura, de manera inmediata la noción de experiencia aparece. Para Anzaldúa, los peligros de escribir acerca de nuestra experiencia son aprendidos; para algunas personas escribir lo que le sucede a las mujeres es trivial, y poco interesante. Por tanto, la política de la escritura 
de la experiencia es el mecanismo del sujeto para defender su perspectiva, el lugar desde dónde observa y vive su formación. Se observa lo que se apetece a partir de un cuerpo. Situar la experiencia es también reconocer las lenguas bajo las cuales las personas dan cuenta de su realidad.

\subsection{Transformar la idea de una experiencia femenina.}

Mirar la experiencia con los lentes del feminismo y de los estudios de género, produce tres consecuencias para desligitimar a la experiencia femenina:

Primera. No existe una noción específica de "lo femenino" que obligatoriamente atribuya un tipo de "experiencia femenina" a las "características" de una mujer biológica. La experiencia del sujeto es también una construcción cuyo fin no es parecerse a otra persona específica, o a un ideal femenino/masculino, a pesar de que la cultura tenga uno prescrito para ambos géneros (omitiendo, como es propio de la cultura heteronormativa, la presencia de personas transgénero e intersexuales).

Segundo. Es imposible "encontrar" una experiencia femenina única. Aquí la pregunta no es por cuál es la experiencia sino cómo a través de la experiencia los sujetos son transformados.

Tercera. La historia tiene un carácter dinámico, de ahí que no sean posibles visiones estáticas acerca del sujeto de la experiencia y que este sujeto no tenga justamente la capacidad de agencia sobre su propia experiencia, la cual, si bien es producto y vigilada por discursos, es como la historia misma: cambiante, aleatoria, discontinua y regenerativa.

Lo femenino agrupa en Occidente a personas y adjetivos: niña, indígena, mestiza, o del tercer mundo, débil, pobre, por lo que entonces, "lo femenino" hace referencia al otro, al que no soy yo.

El sujeto de la experiencia- desde la crítica que se ha intentado desarrollar- no puede ser "mujer" u "hombre" en tanto se estaría prescribiendo una serie de experiencias a un sujeto marcado por su sexo biológico. La experiencia es como el género, una construcción. El género, al ser una construcción social y cultural de la diferencia sexual, es una categoría cultural que emergió para problematizar la diferenciación entre los géneros, no solo desde el componente biológico sino también desde la psiquis y la socialización (Lamas, 2000, p. 2).

Xihmai 40 
El componente cultural visualizado por la categoría de género escapa, entonces, al proyecto de la idea de una experiencia como esencia, o como algo que posiblemente suceda si se cumplen con algunos requisitos. Y escapa, también, a la relativización y la totalización. La experiencia se relativiza cuando se espera un tipo de experiencia para un grupo de sujetos en especial y se totaliza si debe ser igual para todos los sujetos. Tanto la experiencia como el género, entendiéndolos como construcciones, se encuentran del lado de los procesos que dan forma a la identidad de los sujetos y, por esta razón, su estudio debe ser complejo más no relativizante o totalizante.

En consecuencia, la experiencia no se puede situar sobre unos sujetos específicos, ni tampoco puede estudiarse con la ayuda de un método que no sea situado. María Zambrano escribe que el método de la experiencia es su camino mismo, el cual no conduce a algo esperado. Solo existe claridad en algo: la experiencia requiere ser situada (Haraway, 1995), al igual que el género, pues ella sucede al interior de una cultura.

El reconocimiento cultural a la idea de experiencia nos remite con las teorías de la interseccionalidad. Kimberlé Williams en su texto Mapping the Margins: Intersectionality, Identity Politics, and Violence Against Women of Color (1994) argumenta cómo la interseccionalidad da forma a la experiencia de las mujeres de color. Este estudio describe que la experiencia sitúa a los sujetos a través de tres componentes: el género, la raza y la clase (Williams, 1994, p. 3):

At the simplest level, race, gender, and class are implicated together because the fact of being a woman of color correlates strongly with poverty. Moreover, the disparate access to housing and jobs-that is, the phenomenon of discrimination is reproduced through their race and gender identity. Race and gender are two of the primary sites for the particular distribution of social resources that ends up with observable class differences. And finally, once in a lower economic class, race and gender structures continue to shape the particular ways that women of color experience poverty, relative to other groups.

Un nivel simple, el de la pobreza, hace que en el estudio de Williams un tipo de experiencia se le adjudique a un grupo de una población específica: las mujeres. Y este nivel ayuda a comprender (Haraway, 1995, p. 332) que "no hay manera de "estar"" simultáneamente en todas, o totalmente en algunas de las posiciones privilegiadas (subyugadas) estructuradas por el género, la raza, la nación y la clase". 
El trabajo de Anzaldúa abrió el camino para acercarnos a procesos de concienciación situados desde las experiencias, prácticas culturales y discursivas de las chicanas: contó acerca de la necesidad de renunciar a los procesos de dominación aún vigentes en su cultura como acciones de descolonización.

\section{Chorus}

¡Volveremos!

Prenderemos la guerra de bien adentro

como esa luz del alma.

en esta noche Zurda

renacerá el Espíritu

de nuestra Tierra.

Retornará nuestra antigua fe

y levantará el campo.

Arriba, despierta, mi gente

a liberar a los pueblos.

El coro de su canción Arriba mi gente (1987, p. 193) deja leer que la transformación de Anzaldúa es de retorno y de abrir los ojos, sin considerar las expectativas del otro y sin que alguien como su "mamá, la Iglesia, la cultura de los anglos" le señale lo que debe hacer. Gloria Anzaldúa (1987, p.157) abandonó la casa materna "para poder encontrarme a mí misma, encontrar mi propia naturaleza intrínseca, enterrada bajo la personalidad que me había sido impuesta". A diferencia del sujeto de los privilegios que sale de su hogar y recibe de parte de la sociedad las llaves del mundo, el sujeto de la transformación se va de la casa materna y paterna para regresar a ella de visita e incomodar con su rebeldía. A Anzaldúa (1987, p.157) le decían: "muy andariega mi hija. Because I left of my own accord me dicen, ¿cómo te gusta la mala vida?".

De esta manera, la experiencia al no optar por el camino que lleva a una esencia, a la casa materna, opta por el camino sin método. Cargada del simbolismo de la búsqueda que emprende el viajero, la experiencia es el camino de la incertidumbre, pero también el paso a través de la pasión. Sin deseos de caminar hacia lo que no se conoce, no puede ser posible la experiencia. Sin el miedo a encontrar nada al final de las transformaciones en el sendero, tampoco es posible; ni mucho menos cuando se está obligado/a llegar y establecerse en punto de vista ajeno al de la propia experiencia.

Xihmai 42 
La experiencia al no adscribirse a un grupo de personas por razones de género o de sexo, esto es, al no estarnos permitido señalar una experiencia como femenina y otra como masculina, requiere ser interpretada. La experiencia que se deba esperar de un sujeto específico no es la evidencia de una experiencia adjudicada a un grupo.

En últimas, la experiencia es una interpretación de sí.

\section{Fuentes de Consulta}

- ALCOFF, L. (2010). Phenomenology, Post-structualism, and Feminist Theory on the Concept of Experience, En Fisher \& Embree (Eds.). Feminist Phenomenology (pp. 39-56). Netherlands: Kluwer Academic Publishers.

(2002). M. Rosario Martín Ruano (Trad.). Feminismo cultural vs. Post-estructuralismo: la crisis de la identidad de la teoría feminista. Revista Debats, 76, 1-26.

- ANZALDÚA, G. (1988). Hablar en lenguas. Una carta a escritoras tercermundistas. En Moraga \& Castillo (Eds.). Esta puente, mi espalda. Voces de mujeres tercermundistas en los Estados Unidos. (pp.218-222). San Francisco, Estados Unidos: ISM Press.

(1987). (4 ed). Borderlands/La frontera. The New Mestiza. San Francisco, Estados Unidos: Aunt Lute Books.

- $\quad$ DE LAURETIS, T. (1989). Tecnología del género. En Diferencias. Etapas de un camino a través del feminismo. (pp. 33-69). Madrid, España: Horas y horas.

. (1984) 6. Semiótica y experiencia. En Alicia ya no. Feminismo, Semiótica, Cine. (pp. 251-294). Valencia, España: Ediciones Cátedra, Universitat de València, Instituto de la mujer.

- BONDI, L. (1996) M. Lamas (Trad.). Ubicar las políticas de la identidad. Debate Feminista. 7, 14, 14-37. 
- HARAWAY, D. J. (1995). Capítulo 7. Conocimientos situados: la cuestión científica en el feminismo y el privilegio de la perspectiva parcial. En Ciencia, cyborgs y mujeres. La reinvención de la naturaleza. (pp. 313-346). Madrid, España: Cátedra.

- LAMAS, M. (2000). Diferencias de sexo, género y diferencia sexual. Cuicuilco. 7, 18, 1-24.

- SALCEDO, D. (2010) Next: Doris Salcedo, programa Art:21, Canal Film \& Arts. Recuperado el 15 de noviembre de 2015, de: https://www.youtube.com/watch?v=9AAst32Ss7w

- SCOTT, J. (1992). Moisés Silva (Trad.). Experiencia. La ventana. Revista de Estudios de Género, II, 13, 42-73.

- WILLIAMS C., K. (1994). Maping the margins: Intersectionality, Identity Politics, and Violence Against Women of Color. En Alberston Finemam \& Mykitiuk (Eds.). The Public Nature of Private Violence. (pp. 93-118). New York, Estados Unidos: Roudledge.

- WOOLF, V. (2006) (2d.) Un cuarto propio. Santiago de Chile, Chile: Editorial cuarto propio.

- ZAMBRANO, M. (1986). Claros del bosque. Barcelona, España: Editorial Seix Barral, S.A. 